\title{
Norois
}

Environnement, aménagement, société

$201 \mid 2006 / 4$

Dynamiques fluviale et littorale, activité viticole

\section{Le vignoble au Québec, géographie d'un rêve sous contrainte}

The Québec wine industry, geography of a dream against all odds

\section{Hélène Velasco-Graciet et Frédéric Lasserre}

\section{(2) OpenEdition}

1 Journals

\section{Édition électronique}

URL : https://journals.openedition.org/norois/1765

DOI : 10.4000/norois. 1765

ISBN : 978-2-7535-1549-9

ISSN : $1760-8546$

\section{Éditeur}

Presses universitaires de Rennes

Édition imprimée

Date de publication : 1 décembre 2006

Pagination : 67-82

ISBN : 978-2-7535-0407-3

ISSN : 0029-182X

\section{Référence électronique}




\title{
LE VIGNOBLE AU QUÉBEC, GÉOGRAPHIE D’Un RÊVE SOUS CONTRAINTE
}

\author{
HÉLÈNE VELASCO-GRACIET \\ ADES UMR 5185 CNRS \\ (université de Bordeaux 3) - Maison des Suds \\ Esplanade des Antilles - 33607 PESSAC \\ helene.velasco@u-bordeaux3.fr \\ FrÉDÉRIC LASSERRE \\ Département de géographie - université Laval \\ Québec G1K 7P4 (Canada) \\ Frederic.lasserre@ggr.ulaval.ca
}

\section{RÉSUMÉ}

La province du Québec a vu, en moins de dix années, le nombre de ses exploitations viticoles commerciales doubler. Le vignoble québécois peut être caractérisé à la fois de «petit vignoble » par sa superficie et de «vignoble de l'extrême » par les conditions climatiques mais aussi législatives qui le touchent. Malgré ces contraintes difficiles et pesantes que d'aucuns penseraient insurmontables, les vignerons québécois ne manquent ni de ténacité ni de rêve.

Mots CLÉ : Contraintes - Imaginaire géographique - Québec - Vignoble - Vin.

\section{ABSTRACT}

\section{The Québec wine industry, geography of a dream against all odds}

The Province of Québec witnessed a doubling of its commercial vineyards in less than ten years. The Québec wine industry can be described both as centered on "small vineyards" because of the average size of its operations, and as a "vineyard of the extreme" because of the climatic as well as legal conditions that affect it. Despite these difficult conditions that one would deem insuperable, Quebec wine makers do not lack tenacity or fantasy.

KEY WORDS : Constraints - Geographical Imagination - Québec - Vineyard - Wine.

À première vue, s'intéresser à la viticulture du Québec fait figure, en ce début de siècle, de gageure. À l'heure de la crise viticole de ce que certains appellent la nowvelle planète des vins, les chercheurs invitent plutôt à porter le regard sur les mutations sans précédent qui touchent le monde vitivinicole et, qui en le déstabilisant, semblent l'entraîner vers une situation d'anomie. La difficulté pour cerner cette crise est le choix de l'entrée à opérer. En effet, l'ensemble des strates de la filière connaît des mouvements indépendants les uns des autres mais qui viennent se percuter et se nourrir entre eux, accélérant ainsi le processus global en cours. Ainsi, la pluralité des productions, la multiplicité des goûts, la féminisation des clientèles, l'émergence de nouvelles pra- 
tiques du vin imbriquées dans de nouveaux rapports à l'aliment au sein de cultures et d'habitudes alimentaires très éloignées de celles de la vieille Europe, la diversité des techniques et des procédés de fabrication du vin, l'accélération des échanges mondiaux, sont autant de causes explicatives et d'autonomie partielle. En fin de compte, nous ne retiendrons de cette crise qu'une seule chose : les divers mouvements économiques, sociaux et culturels contemporains remettent aujourd'hui en cause un ordre mondial viticole forgé, avec force et patience, dès le début du XIX siècle. Cet ordre se fondait sur une hiérarchie territoriale extrêmement précise prenant appui sur deux notions liées et, en ce qui concerne le vin, mondialement partagées : la qualité et la distinction sociale. Dégagés d'une sorte de carcan, des vignobles naissants ou renaissants à travers le monde apparaissent aujourd'hui, augmentant les inquiétudes et les incertitudes de celles et ceux qui voudraient encore s'inscrire dans un continuum. Les vignobles québécois sont de ces nouveaux vignobles et, à leur façon, participent à la refondation d'une nouvelle planète des vins.

Le vignoble québécois n'est pas le seul au Canada. Les vignobles luxuriants de l'Ontario et la Colombie-Britannique produisent la majorité des raisins de l'industrie vinicole canadienne. Ensemble, ces deux provinces fabriquent $98 \%$ du volume de vins de marque du pays. Le Québec et la Nouvelle-Écosse ont développé de petits vignobles, réduits mais aux exploitants enthousiastes.

Il est difficile de définir le vignoble québécois. Si J.-P. Augustin (1993) en le qualifiant d'a-historique, lui retire un passé et donc une mémoire, au contraire R. de Koninck (1993), en s'appuyant sur les récits des premiers explorateurs, propose une version tout autre. La vigne serait endémique à la contrée et la viticulture québécoise aurait connu moult rebondissements, toute une succession de réussites et d'échecs qui témoignerait « d'une détermination farouche... de tous ceux qui, depuis le début de la «colonie », se sont entêtés à faire pousser de la vigne en ce pays de neige et de froidure » (de Koninck, 1993). À ce titre, on peut qualifier ce vignoble de « vignoble du défi » en se référant à la perspicacité de ces promoteurs, à leur audace et à leur entêtement, grâce auxquelles ils auraient réussi à cultiver de la vigne bien au-delà des « frontières climatiques que la nature semblait lui interdire de dépasser»(Dion, 1990). Plusieurs auteurs se sont déjà penchés sur le paradoxe apparent de la culture de la vigne au Québec, sous un climat peu propice et dans une société qui, pour en avoir consommé sous le régime français, est devenue largement consommatrice de bière plutôt que de vin pendant longtemps : mentionnons ainsi les travaux, parfois vieillissants mais fondamentaux, de G. Masson (1983), R. De Koninck (1992 et 1993), F. Lasserre (2001), et surtout de L. Deshaies et J.-M. Dubois (1993, 1997a, 1997b), L. Deshaies (2003), J.-M. Dubois (1996), par ailleurs auteurs de plus d'une vingtaine de publications sur le développement du vignoble québécois et ses contraintes climatiques et commerciales. Cependant, peu détaillent la dimension sociologique de la production de vin : qui sont ces producteurs? Pourquoi se lancent-ils dans un pari aussi risqué commercialement?

On parle aussi de «petit vignoble » au Québec, au regard tant du nombre d'exploitations que de la superficie plantée ou du volume de la production et ce, malgré un très vif essor depuis une vingtaine d'années (tableaux 1 et 2).

\begin{tabular}{|c|c|c|c|c|c|c|c|c|c|}
\hline $\begin{array}{c}\text { États- } \\
\text { Unis }\end{array}$ & Canada & Québec & Argentine & Chili & Australie & $\begin{array}{c}\text { Afrique } \\
\text { du sud }\end{array}$ & France & Italie & Espagne \\
\hline 19800 & 760 & 4,1 & 13500 & 6700 & 13900 & 6400 & 48000 & 49500 & 40300 \\
\hline
\end{tabular}

Tableau 1 : Production comparée de vin du Québec, du Canada et des autres producteurs majeurs mondiaux (chiffres en milliers d'hectolitres) (Sources : Organisation internationale de la vigne et du vin [OIV], 2003; Statistiques Canada, 2006; Vitisphère, 2006; Québec : estimation des auteurs sur compilation des données des vignobles en 2005)

toliters)

Compared wine production in 2005 : Québec, Canada and other major producers (million hec- 


\begin{tabular}{|c|c|c|c|c|c|c|c|c|c|}
\hline $\begin{array}{c}\text { États- } \\
\text { Unis }\end{array}$ & Canada & Québec & Argentine & Chili & Australie & $\begin{array}{c}\text { Afrique } \\
\text { du sud }\end{array}$ & France & Italie & Espagne \\
\hline 389 & 8,84 & 0,33 & 208 & 168 & 150 & 121 & 900 & 850 & 1200 \\
\hline
\end{tabular}

Tableau 2 : Superficie comparée des vignobles au Québec, au Canada et dans les autres producteurs majeurs mondiaux (chiffres en milliers d'hectares) (Sources : Vitisphère, 2006; Statistiques Canada, 2006; Québec : compilation des données des vignobles en 2005)

Compared vineyard areas in 2005: Québec, Canada and other major producers (thousand hectares)

En effet, on recense 66 vignobles commerciaux en activité en 2005, contre 33 en 1998. La superficie totale exploitée est de 330 ha, soit en moyenne 5 ha par exploitation, avec des extrêmes de 20,5 ha pour la plus grande et de 1,25 ha pour la plus petite. Les productions sont peu importantes, de l'ordre de quelques milliers de bouteilles; la plus importe d'entre elles s'élève à 100000 bouteilles, au vignoble du Cep d'Argent. Cependant, avec la progressive maturation des vignes, pour la plupart plantées au cours de la décennie 1990, la production globale a connu un accroissement rapide, passant de 303300 bouteilles en 1995 à 528700 en 1999, soit une augmentation annuelle moyenne de l'ordre de 14,9\%. Mais ces volumes sont faibles en regard de la consommation globale de vin et les vins québécois produits sur ces vignobles ${ }^{1}$ ne représentaient que 0,5\% du marché du vin au Québec en 2003. Si la viticulture est concentrée dans les régions de Montérégie et d’Estrie; on recense quelques vignes dans la région de Québec et en Lanaudière (fig. 1).

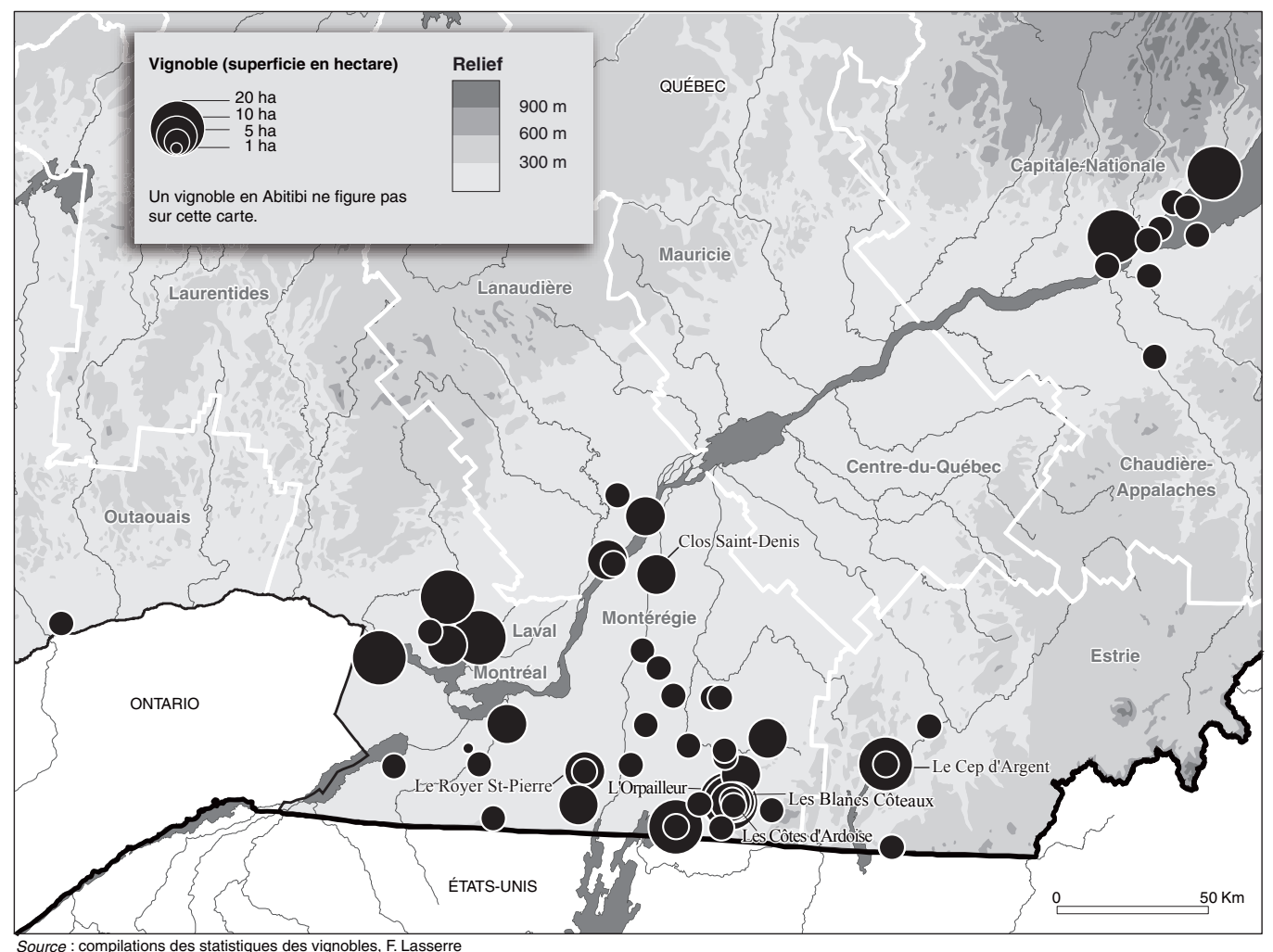

Figure 1 : Les vignobles commerciaux du Québec en 2005

Commercial vineyards in Quebec, 2005

1. Par opposition à des vins élaborés à partir de raisin importé, voire de jus concentré. 
Si à l'échelle mondiale, le vignoble québécois et sa production sont anecdotiques, à l'échelle provinciale, son rapide développement depuis un peu plus de vingt ans invite à s'interroger. Il ne s'agit bien sûr pas de procéder à une analyse par analogie avec un « grand vignoble » qui par sa taille et sa production se place sur le marché mondial en se soumettant au système concurrentiel international : le vignoble québécois ne se compare en rien aux vignobles chiliens, argentins, australiens ou sud-africains, très compétitifs sur les marchés internationaux, produits en grandes quantités et dans des grands domaines (Velasco-Graciet et Hinnewinkel, 2005), ou même de l'Ontario et de la Colombie-britannique au Canada. Le développement du vignoble québécois obéit, en effet, à d'autres logiques que nous tenterons ici de mettre en évidence. En fin de compte, l'interrogation qui traverse cet article est : pourquoi cultiver de la vigne et produire du vin alors que tant de contraintes objectives inviteraient à renoncer d'emblée? On notera, en premier lieu, une contrainte climatique quasi insurmontable qui nécessite le recours à des stratégies et des techniques difficiles et onéreuses influençant de façon notable le prix de revient par bouteille et interdisant, sauf dans de très rares exceptions, la plantation de cépages nobles. À cette contrainte « naturelle » s'additionnent une contrainte législative et administrative qui par l'intermédiaire de la Société des Alcools du Québec (SAQ) empêche une distribution aisée de la production et une contrainte culturelle qui fait que ni la culture de la vigne ni la consommation de vin ne font partie d'un corpus de savoir et de savoir faire commun.

Alors que tout pousserait donc à une stagnation ou à un renoncement, la réalité de la viticulture québécoise, en se développant, montre tout autre chose : un petit vignoble se défiant des contraintes climatiques, législatives et culturelles. En fin de compte, dans le désordre que connaît le monde vitivinicole, le vignoble québécois possède une place même s'il est mu par d'autres logiques que des logiques économiques et capitalistes. Il se rapprocherait de ce que P. Roudié (1989) a nommé les « vignobles de l'extrême » et qui bénéficie aujourd'hui d'une image positive auprès de consommateurs en quête de nouveautés et d'expériences (même gustatives) uniques. Car bien sûr, si boire du vin est bien plus qu'un simple acte alimentaire, le produire relève d'un processus complexe se situant au-delà de la production agricole. Finalement, après avoir décliné les diverses contraintes et dessiné une géographie du vignoble québécois, nous tenterons de répondre à la question : quel est le sens d'une production de vin au Québec?

\section{Le vignoble québécois, un vignoble sous contrainte}

\section{LES CONDITIONS CLIMATIQUES : IMPACTS ET RÉPONSES}

La culture de la vigne n'est pas nouvelle au Québec : des espèces rustiques locales, adaptées à des températures de l'ordre de $-30^{\circ} \mathrm{C}$, étaient cultivées pour une production domestique, voire pour l'autoconsommation. À partir de 1860, il s'était créé une trentaine de vignobles dans le sud du Québec, sur une superficie exploitée de l'ordre de 40 ha. En 1901 il y avait encore 109000 plants de vigne répartis sur 94 fermes. En 1935, du fait de l'impact de la prohibition, de l'évolution des goûts et de la pression vers une plus forte rentabilité induite par la dépression économique, la superficie de l'ensemble des vignobles, commerciaux ou non, était inférieure à 2 ha (Masson, 1983). C'est en 1977 qu'apparaissent au Québec les premiers vignobles commerciaux (avec permis de vente). En 1984, on comptait 128000 pieds de vigne, et plus de 500000 en 2000.

Tous les vignobles du Québec cultivent des cépages hybrides de cépages européens et nordaméricains; depuis une quinzaine d'années, certains, avec grands soins, ont introduit des cépages de Vitis vinifera, la variété de vigne européenne, comme le geisenheim, le chardonnay, le bacchus, le gamay. Ces choix en faveur de cépages hybrides résultent, bien évidemment, des contraintes climatiques : les cépages qui permettent d'envisager l'exploitation d'un vignoble au Québec doivent s'accommoder d'une saison végétative courte, assurer une maturation accélérée de la vigne, et bien sûr pouvoir résister au froid de l'hiver. 
La latitude de la frontière méridionale du Québec, à environ 60 km de Montréal, est la même que celle de Bordeaux, et Québec est à la même latitude que la Côte-d'Or bourguignonne. Ce n'est donc pas un critère adéquat pour déterminer les contraintes climatiques à la culture de la vigne. La vigne se contente de peu d'eau, ce ne sont donc pas les précipitations qui sont déterminantes au Québec, mais plutôt les températures hivernales. A priori, les conditions sont plutôt défavorables (tableau 3). La température annuelle moyenne de la plaine à l'Est de Montréal est de $6{ }^{\circ} \mathrm{C}$, alors que la vigne ne tolère pas, normalement, une température moyenne inférieure à $9{ }^{\circ} \mathrm{C}$. De même, les bourgeons meurent en général lorsque le gel descend sous la barre des $-25^{\circ} \mathrm{C}$, mais il arrive à l'occasion que le froid atteigne des valeurs inférieures, jusqu'à $-37^{\circ} \mathrm{C}$ dans le sud du Québec. Enfin, la saison végétative est nettement plus courte, avec des températures moyennes pour avril qui empêchent la vigne de croître rapidement au début du printemps. Mais l'insolation estivale est comparable, et la chaleur humide de l'été québécois arrive à compenser, avec des cépages à pousse rapide, la brièveté de cette saison de croissance, qui débute en avril avec le débuttage des ceps, enterrés pour mieux résister au froid, et s'achève avec des vendanges vers la mi-septembre. La vigne peut avoir débourré fin avril ou début mai, alors que la probabilité d'un gel nocturne est encore de l'ordre de 75 \% (Dubois et Dehaies, 1997).

Des techniques particulières permettent aux pieds de vigne de supporter le froid hivernal, en particulier le renchaussage des pieds après la taille, début novembre généralement ; les pieds sont débuttés au printemps, fin avril ou début mai, mais le vigneron doit estimer correctement la date de l'opération pour se prémunir des gelées printanières tardives. Ces opérations demandent une adaptation des tracteurs aux outils spécifiques à ces opérations délicates.

La faible quantité de neige, certains hivers, permet au froid de pénétrer dans le sol. Certains vignobles ajoutent donc un lit de feuilles mortes ou de paille pour pallier cette éventualité; d'autres envisagent de s'équiper avec des canons à neige, comme pour les stations de ski, une possibilité que renforce la récurrence d'hivers avec très peu de neige depuis quelques années. Enfin, certaines exploitations se sont équipées de brûleurs disposés en des endroits propices pour faire face à des chutes brutales de température. On a même vu quelques vignobles recourir à des hélicoptères pour rabattre l'air chaud vers le sol (Lasserre, 2001).

\begin{tabular}{|c|c|c|c|c|c|}
\hline $\begin{array}{l}\text { Station } \\
\text { météorologique }\end{array}$ & $\begin{array}{c}\text { Plus basse } \\
\text { température } \\
\text { enregistrée, }{ }^{\circ} \mathrm{C}\end{array}$ & $\begin{array}{c}\text { Température } \\
\text { moyenne, } \\
\text { janvier, }{ }^{\circ} \mathrm{C}\end{array}$ & $\begin{array}{c}\text { Température } \\
\text { moyenne, avril, } \\
{ }^{\circ} \mathrm{C}\end{array}$ & $\begin{array}{c}\text { Degrés-jours } \\
\text { au-dessus de } \\
10{ }^{\circ} \mathrm{C}\end{array}$ & $\begin{array}{c}\text { Heures } \\
\text { d'insolation, } \\
\text { avril-septembre }\end{array}$ \\
\hline Montréal & $-37,8$ & $-10,3$ & 5,7 & 1215 & 1320 \\
\hline $\begin{array}{l}\text { Philipsburg } \\
\text { (Montérégie) }\end{array}$ & $-37,6$ & $-10,2$ & 5,6 & 1200 & 1366 \\
\hline $\begin{array}{l}\text { Saint-Jean sur } \\
\text { Richelieu } \\
\text { (Montérégie) }\end{array}$ & $-37,8$ & $-10,2$ & 5,4 & 1029 & 1269 \\
\hline $\begin{array}{l}\text { Rougemont } \\
\text { (Montérégie) }\end{array}$ & $-37,8$ & $-10,4$ & 5,4 & 1022 & 1274 \\
\hline $\begin{array}{l}\text { Lennoxville } \\
\text { (Estrie) }\end{array}$ & $-38,3$ & $-11,6$ & 3,9 & 883 & 1254 \\
\hline Québec & $-35,4$ & $-12,4$ & 3,3 & 861 & 1255 \\
\hline Bordeaux & $-15,1$ & 5,8 & 11,3 & 1403 & 1375 \\
\hline Dijon & $-20,2$ & 1,6 & 9,8 & 1150 & 1308 \\
\hline Nantes & $-16,1$ & 5,4 & 10,4 & 1157 & 1342 \\
\hline Strasbourg & $-21,2$ & 0,9 & 9,6 & $\mathrm{Nd}$ & 1192 \\
\hline
\end{tabular}

Tableau 3 : Comparaison des conditions climatiques pour quelques localités québécoises et françaises (source : Environnement Canada, 1998; Météo France, 1999)

Compared climatic conditions for several points in Québec and France 
Ces contraintes climatiques ont orienté les choix des vignerons vers des ceps rustiques. La culture de nombreux cépages est très courante, jusqu'à une douzaine pour certaines exploitations. La moyenne se situait en 1999 à 5,1 variétés principales ${ }^{2}$ différentes par vignoble, à 5,4 en 2005 . Cette diversité procède en partie des efforts d'expérimentation et de recherche des cépages offrant la meilleure qualité compte tenu des contraintes du climat. Une autre raison réside dans la pratique courante de l'assemblage, destiné soit à expérimenter des goûts différents, le plus souvent à compenser les faiblesses des récoltes de certains cépages certaines mauvaises années. Environ $85 \%$ des vins québécois sont des assemblages. Le seyval, le cépage le plus répandu et avec lequel on produit l'essentiel des vins blancs de cépage, s’il ne permet pas de produire des crus exceptionnels, rend possible la création d'un vin léger et très acceptable lorsqu'il est vinifié avec soin. La viticulture québécoise est encore en évolution, avec une forte dimension de recherche, tant des meilleurs cépages que des meilleures techniques de vinification. En revanche, d'autres vignobles mettent à profit ces conditions climatiques pour tenter de faire des vins de glace, ou vins de vendanges tardives, sur le modèle des ice wines, des vins de glace ontariens ou allemands. Le Canada est reconnu comme le premier fabricant de vins de glace au monde en volume (120000 litres exportées en 2004) et continue de recevoir des médailles d'or dans les plus prestigieuses compétitions internationales (Hope-Ross, 2006).

Ainsi, certains cépages dominent les vignobles québécois, car il s'est progressivement avéré que ceux-ci convenaient mieux aux conditions climatiques du Québec. Les cépages blancs, plus adaptés aux contraintes climatiques, dominent : le vin blanc représente plus de $55 \%$ du volume total de la production, (y compris les produits dérivés, apéritifs, digestifs, assemblages avec d'autres alcools), et $70 \%$ du volume des vins.

Les contraintes climatiques influencent également les techniques de vinification. À l'instar des récoltes allemandes ou autrichiennes, la production de raisins au Québec manque souvent d'un peu de maturité, ce qui donne des jus présentant une forte acidité. La fermentation malolactique est alors très souvent (dans $90 \%$ des cas) volontairement décalée de la fermentation alcoolique, car le froid de l'hiver permet une réduction de l'acidité, ce qui facilite le lancement des fermentations malolactiques vers la fin de l'hiver. Dans les années 1980, la tendance était au blocage complet de la fermentation malolactique, mais les goûts des consommateurs québécois, à la différence d'une tendance marquée aux États-Unis, se sont éloignés des vins vifs, pour préférer les vins plus tendres que permet cette fermentation. Par ailleurs, $95 \%$ des vins québécois sont chaptalisés pour compenser la faible maturité des récoltes.

\section{Des SOlS PROPICES MAIS PEU VARIÉS}

Les vignobles de Montérégie, de l'Estrie sis sur le piémont des Appalaches, ou de la région de l'île d'Orléans, sont cultivés sur des sols relativement propices à la culture de la vigne, mais ils présentent des variations assez faibles, ce qui ne favorise pas la différenciation des goûts. Plus de $50 \%$ des vignobles ont un sol dominé par les argiles ou les sables silteux, une forte proportion (environ $25 \%$ ) par des sables avec graviers plus ou moins fins; ils se concentrent dans la plaine de Montérégie et de l'ouest de l'Estrie. Les vignobles du piémont des Appalaches sont des tills plus acides dus à la présence d'argiles de désagrégation de micaschistes et de schistes. Rares sont les sols présentant des $\mathrm{pH}$ basiques, comme celui du vignoble de l'Aurore boréale, composé de sables et de loam calcaire. Le pH souvent acide oblige les exploitants à régulièrement amender les sols avec de la chaux, du bore ou du magnésium.

\section{LES CONDITIONS DE DISTRIBUTION : POIDS ET ESPOIR}

Un obstacle majeur à l'expansion du vignoble québécois a longtemps résidé dans la difficulté d'écouler les productions. La Régie des alcools du Québec (RAQ), qui contrôle la vente des pro-

2. Hors expérimentation, dont la récolte n’est pas intégrée dans la production commerciale. 
duits alcoolisés sur le territoire provincial, a longtemps octroyé avec parcimonie les permis de vente sur le site de production. Les difficultés d'ordre commercial ne se sont pas arrêtées là : la Société des Alcools du Québec (SAQ), société d'État et principal instrument de la distribution des vins au Québec, a longtemps refusé d'assurer la mise en marché de la production des vignobles québécois. Encore aujourd'hui, de nombreux vignobles commerciaux n'ont pas pu signer de contrat de distribution auprès de la SAQ, qui préfère s'en tenir aux crus étrangers qui offrent des volumes plus importants et des qualités plus constantes : en 2004, les vins québécois représentaient $0,1 \%$ de ses ventes de vins (Association des Vignerons du Québec, 2005) 3. À partir de 1996, la SAQ s'est efforcée, sous la pression des associations de vignerons et du ministère de l'Agriculture, de développer des formules de distribution qui pourraient lui permettre de concilier les faibles volumes de production des vignobles québécois, et ses propres contraintes qui la conduisent à favoriser les gros volumes. En 2005, la SAQ avait ainsi accepté l'idée de créer une section des vins du Québec dans ses succursales. Il faut dire que les goûts des consommateurs changent : si le Québec et le Canada demeurent des pays de bière, en 2005, pour la première fois la valeur des ventes de vin (25,2 \% du marché de l'alcool) a dépassé celle des alcools forts $(24,3 \%)$, avec la bière représentant $50,4 \%$. C'est au Québec que les consommateurs achètent le plus de vin, avec 18,2 litres par adulte en 2005, suivi de la Colombie-Britannique avec 16,2 litres (Hope-Ross, 2006). Le consommateur québécois est désireux d'essayer de nouveaux vins, ce qui favorise son intérêt tant pour les vins québécois qu'étrangers, mais ce qui en fait un client à la fidélité relative (Manivet, 2005).

Cependant, l'attitude de la SAQ n'est pas seule en cause dans la difficulté qu'éprouvent les vignobles à distribuer largement leurs produits. Le faible volume produit par chaque vignoble et les conditions difficiles de culture impliquent des prix de vente au domaine de l'ordre de 10 \$ minimum la bouteille (environ 8 euros). Une fois mis en vente dans les réseaux de distribution, foires, marchés publics, restaurants, les vins sont majorés de la marge du distributeur et des taxes fédérale et provinciale. Les vins québécois se trouveraient de fait, comme à la fin du XIXe siècle, peu capables de concurrencer les vins européens, américains, latino-américains ou australiens lorsqu'ils se trouvent mis en vente à près de $17 \$$ minimum la bouteille dans les réseaux commerciaux de la SAQ, soit plus que de nombreux crus étrangers de qualité comparable ou meilleure.

\section{Géographie des vignobles québécois, une géographie autant climatique... que culturelle et commerciale}

\section{ORIGINE SOCIALE ET IMPLANTATION DES VITICULTEURS}

La majorité des propriétaires qui ont fondé ces vignobles québécois ne sont pas, à l'origine, des agriculteurs. On y trouvait un fort contingent de puéricultrices, infirmières, ingénieurs, plombiers, professeurs d'université ou d'arts martiaux, journaliste, travailleurs sociaux; bref, de nombreux citadins qui avaient établi un vignoble non loin de leur ville, Québec ou la région urbaine de Montréal. Leur exploitation a parfois même commencé comme un passe-temps alors même qu'ils étaient encore citadins. C'est un intérêt marqué pour le vin, la vinification, parfois suscité par un voyage en Europe ou une rencontre avec des vignerons européens, beaucoup plus qu'une quelconque philosophie de « retour à la terre », qui anime ces amateurs qui décident de faire du vignoble leur nouvelle activité. Ce sont souvent des amateurs enthousiastes, à l'image du défunt propriétaire du vignoble Dietrich Joss, qui s'est révélé, au cours du temps, être un véritable pionnier par les expérimentations diverses qu'il initia. De fait, la production a souvent d'abord été à usage privé avant de devenir une activité commerciale. Il se greffe parfois, sur cet intérêt pour la chose du vin, un certain militantisme en faveur de la production des produits agricoles au Québec (Dubois et Deshaies, 1997). 
La création de vignobles commerciaux s'accélère depuis 1992: de 11 vignobles en activité en 1989, on passe à 21 fin 1992, puis à 28 fin 1995; 34, fin 1997, 38 en $2000^{4}$ puis 66 fin 2005. On observe une relative concentration géographique en Montérégie et dans l'ouest de l’Estrie, qui s'explique par trois facteurs essentiels :

- un facteur climatique tout d'abord : la Montérégie, au sud-est de Montréal, offrent des conditions acceptables pour les hybrides retenus, avec les techniques de culture développées. Les collines des contreforts occidentaux des Appalaches, en Estrie, en particulier dans la région de Dunham, bien exposées et moins sujettes au gel que la plaine juste à l'ouest, présentent des qualités fort intéressantes tant du point de vue climatique que pédologique.

- Cependant, ce facteur, de même que l'impact des qualités du terroir, a eu un rôle moins important que ce qu'on pourrait supposer, en témoigne l'absence relative du développement de vignoble au nord de la Montérégie, pourtant favorable. En effet, en 1996, 70 \% des propriétaires, souvent urbains, on l'a vu, possédaient leur terrain avant de prendre la décision de monter un vignoble. Le facteur climatique joue donc a posteriori parmi les propriétaires tentés par la viticulture (Dubois et Deshaies, 1997). La proximité des centres urbains est donc importante, car c'est souvent à distance raisonnable de leur ville que nombre de propriétaires ont acheté un terrain à la campagne. La géographie des vignobles recoupe donc en partie celle des espaces de proximité et de villégiature des citadins, ce qui explique l'apparition de vignobles dans la région de Québec, où la saison végétative est encore plus courte qu’en Montérégie.

- L'importance de la localisation près de Montréal et de Québec joue surtout du fait de la relative difficulté d'assurer une distribution de produits qui restent relativement onéreux. Coupé des réseaux de la SAQ et des épiceries, le vigneron doit développer lui-même sa clientèle. Si l'on excepte quelques vignobles qui ont pu développer une mise en marché par la SAQ et les marchés publics, il est donc nécessaire pour la plupart d'entre eux de trouver des débouchés parmi deux marchés cruciaux pour les vignobles québécois :

- la clientèle des touristes et des visiteurs réguliers, donc ceux des fins de semaine, une clientèle essentiellement urbaine;

- le marché de la restauration, soit dans les grandes agglomérations, soit sur place en conjonction avec le développement de la clientèle touristique.

De fait, l'île d'Orléans, la Montérégie et l'Estrie font partie de marchés d'acheteurs : en particulier, ces deux dernières régions, à moins d'une heure d'autoroute de Montréal, bien dotées en attraits touristiques, misent ouvertement sur un tourisme de fidélisation orienté vers les Montréalais. Ainsi l'Association des Vignerons du Québec a-t-elle élaboré des itinéraires touristiques dits de la « route des vins » qui attirent beaucoup de visiteurs à l'automne; de nombreux vignobles offrent des services complémentaires aux touristes, comme de la restauration de qualité, de l'hébergement, de l'élevage d'animaux peu courants (lamas, sangliers, cerfs japonais appelés shikas, yaks, vaches écossaises) pour cibler le public familial. Le ministère du Tourisme a soutenu les vignobles dans leur développement, travaillant en partenariat avec eux pour définir les tracés des "routes des vins », vantés désormais par des brochures dans tous les bureaux de tourisme. Le ministère a vu dans ce nouveau produit touristique un attrait pour séduire une clientèle plus aisée et spécifiquement destinée aux régions rurales; les exploitants y ont trouvé un outil de développement d'une clientèle prompte à s'intéresser aux nouveaux produits du terroir et à se fidéliser. De ce point de vue, le développement des vignobles s'intègre parfaitement dans la tendance d'une partie monde agricole québécois à opter pour une diversification marquée, en optant pour des cultures ou des élevages exotiques, ou en organisant des activités agrotouristiques inédites ${ }^{5}$.

4. Deux vignobles, Saint-Alexandre et Riches-Lieux, ont fermé en 1999.

5. En 1998, environ un millier d'exploitations avaient opté pour ce créneau particulier, soit $3 \%$ des fermes du Québec. Laurent Deshaies, "L'insertion territoriale sur la base d'un projet identitaire : les nouveaux agriculteurs », communication lors du Congrès de l'ACFAS, Montréal, 14 mai 1997. 


\section{LA FRAGILITÉ DES EXPLOITATIONS}

Si quelques exploitants sont aujourd'hui des professionnels formés aux techniques agricoles ou issus de familles d'exploitants agricoles, les vignobles sont, le plus souvent, le fait de citadins, on l'a vu, qui ont d'abord acheté une terre pour leur résidence secondaire, avant d'y envisager l'implantation d'un vignoble. En réalité, on compte une cinquantaine d'autres petits vignobles au Québec, vignobles non commerciaux la plupart de temps exploités par des citadins comme passe-temps.

De fait, les exploitants disposent souvent d'une autre source de revenus, à tout le moins pendant la période d'essai et de rodage. C'est que l'exploitation commerciale d'un vignoble nécessite des investissements coûteux et implique des frais d'exploitations importants tout au long de l'année. La plupart des fermetures de vignobles sont liées à l'impossibilité, pour l'exploitant, d'assumer les coûts qu'implique une exploitation commerciale de son vignoble. La difficulté de l'exploitation ne contribue pas à engager les exploitants à étendre considérablement la surface de leur exploitation, souvent petite, on l'a vu, tandis que l'absence d'une tradition vinicole implique que le financement des caisses coopératives rurales n'est pas facilement mobilisable (Lasserre, 2001).

Quelques vignobles produisent et vendent suffisamment pour permettre à leurs exploitants de vivre de cette activité. Ils ont développé une certaine renommée depuis plusieurs années : le Cep d'Argent, les Arpents de Neige, les Côtes d'Ardoise; l'Orpailleur et Dietrich-Jooss (étudiés par R. De Koninck, 1992, 1993). Bon nombre d'autres exploitants ont comme objectif, à terme, de ne dépendre financièrement que de leur exploitation; il leur faut pour cela atteindre un volume minimal et une certaine régularité dans la production, afin de couvrir les frais fixes et l'amortissement des investissements initiaux, et surtout de conserver une clientèle, qu'il s'agisse d'amateurs ou de restaurants, très sollicitée et facilement échaudée par des expériences négatives. Pour la plupart des exploitants, une source complémentaire de revenus est nécessaire dès lors que l'exploitation n'est pas effectuée à titre d'activité de loisirs du propriétaire. Ainsi, des vignobles comme Les Bauges ou Le Clos de la Montagne élèvent des animaux exotiques. D'autres exploitants possèdent aussi un verger, une culture plus industrielle au Québec et qui assure des revenus réguliers; certains en profitent pour élaborer, parallèlement au vin, des produits alcoolisés dérivés de la pomme, apéritifs doux, cidre, liqueur vinifiée, etc., parfois en faisant preuve de beaucoup d'imagination! D'autres vignobles, enfin, comptent sur des revenus liés à la restauration sur place et aux visites touristiques (Lasserre, 2001). Mais la précarité financière de ces exploitations explique le démantèlement du vignoble Dietrich-Jooss en 2003, à la mort de l'exploitant : personne ne voulait reprendre une exploitation perçue comme encore fort risquée.

\section{LA FRAGILITÉ DE LA DEMANDE}

Si les habitudes de consommation du public québécois en matière de gastronomie ont rapidement évolué, suscitant, en l'espace d'une décennie, une demande nouvelle pour des fromages fins, un choix plus diversifié de vins du monde entier, des bières plus raffinées, et ont ainsi rendu possible l'apparition d'un intérêt du public pour les vignobles québécois, il importe que ceux-ci prennent garde au contrecoup possible de cette évolution du marché. On peut espérer qu'avec le développement de leur savoir-faire en matière de vinification, des crus au goût un peu acide prendront de la texture, et amélioreront ainsi leur rapport qualité/prix. Il n'en reste pas moins que la production québécoise est très inégale. À côté de vins trop jeunes ou sans beaucoup de corps, on trouve quelques crus très acceptables compte tenu des conditions de production et de la jeunesse de la tradition vinicole québécoise. En revanche, les vins québécois sont, de fait, à qualité comparable, nettement plus chers que leurs concurrents européens, australiens ou des Amériques. Leurs prix reflètent les modestes dimensions d'exploitations qui restent essentiellement marginales dans le paysage agricole québécois, dimensions modestes qui ne permettent pas d'économies d'échelles qui seraient pourtant d'autant plus nécessaires que les coûts de revient sont supérieurs à ceux des vignobles européens ou américains. 
On touche là un élément incontournable : les vignobles québécois sont peu rentables. Les investissements sont importants en équipement de lutte contre le froid, les coûts d'exploitation sont plus élevés du fait des multiples opérations de protection de la vigne, les parcelles sont petites. Si l'on peut tirer environ 3 à 4 bouteilles d'un plant de vigne mature en Europe, un plant ne donne qu'un peu plus d'une bouteille au Québec (MAPAQ, 2000).

Pour l'instant, cette faible rentabilité ne semble pas un handicap à leur développement, car les exploitations ont su trouver des marchés dans une clientèle intéressée par le développement d'un produit non seulement nouveau, mais surtout local et porté par l'évolution des goûts et de la consommation : la dimension affective est très importante dans la décision d'achat des consommateurs. Cet engouement procède essentiellement de la diffusion relativement récente de modèles de consommation européens, d'une part, et de l'intérêt de citadins pour les productions artisanales et originales des campagnes québécoises : acheter du vin du Québec constitue donc aussi une activité touristique et ludique. Mais de fait, le développement des vignobles demeure vulnérable : il n'est pas certain que les consommateurs accepteront durablement de parcourir plusieurs dizaines de kilomètres pour acheter de nombreuses caisses d'un produit cher, encore en maturation, et moins raffiné que les vins européens à prix égal. Pour que sa qualité s'améliore, il est nécessaire de procéder à d'importants investissements que les vignobles ne peuvent guère financer; de plus, leur niveau de prix ne permet guère d'espérer une augmentation de leurs marges du côté des consommateurs actuels.

L'existence des vignobles du Québec reflète donc avant tout une relation entre citadins et monde rural : elle procède d'une évolution des goûts gastronomiques de ces citadins que vient relayer le souci de consommer davantage de produits québécois, d'une certaine fierté de défier ainsi les conditions climatiques pour parvenir à produire un vin acceptable, et du désir de certains citadins de se lancer dans la production d'une denrée agricole à fort contenu culturel. Ces facteurs socioculturels, qui impliquent une faible diffusion de projets de vignobles dans le monde rural (à l'heure actuelle du moins), et les contraintes économiques font qu'à moyen terme, il est peu probable que le vignoble québécois devienne autre chose que ces sympathiques petites exploitations, sans prétention et sans poids économique agricole majeur. En revanche, elles deviennent des atouts touristiques non-négligeables. Mais il s’agit désormais d'une autre logique économique.

\section{Ce que faire du vin au Québec veut dire}

Cette troisième partie s'appuie sur une série d'entretiens menés au cours de l'été 2004 auprès de viticulteurs. Sur les vingt-huit viticulteurs recensés par l'Association des Vignerons du Québec comme participants à la Route des vins du Québec, vingt d'entre eux ont été visités dans trois des cinq régions viticoles que compte le Québec : les régions des Basses Laurentides, de Montérégie et de l'Estrie (Cantons de l'Est) ${ }^{6}$.

Cette méthode apparaît opportune pour plusieurs raisons liées. La jeunesse de la viticulture commerciale au Québec nous a, en effet, permis de rencontrer dans $80 \%$ des cas des exploitants premiers planteurs et pour les $20 \%$ restants, il s'est agi de repreneurs de première main. Ainsi, dans les deux cas, les personnes rencontrées sont celles qui ont initié le processus ou ont connu directement les premiers planteurs. Par l'intermédiaire des entretiens, nous avons pu cerner dans la logique des discours mis en place, les motivations premières, les stratégies et les expérimentations de chacun de nos interlocuteurs. Plus que des entretiens classiques, il s'agit plutôt d'histoires de vie ou d'histoires de «tranches » de vie. Ainsi, par cette méthode, nous avons pu " accéder (par l'intérieur) à une réalité qui dépasse le narrateur et le façonne » (Balandier, 1983). Ces histoires nous ont permis de cerner la genèse du vignoble québécois, son récent et encore modeste développement, de déceler les rêves et les motivations de ses promoteurs. 


\section{LE RÊVE DE CULTIVER DE LA VIGNE ET DE PRODUIRE DU VIN}

Le contexte général est bien sûr à prendre tout d'abord en compte pour expliquer ce phénomène qui, il y a quelques années, n'était absolument pas envisageable dans sa forme commerciale et, à ses débuts dans les années 1980, relevait de la gageure : "Quand on a planté des vignes ici, les gens nous regardaient comme si on avait perdu la tête, c'était tordant ${ }^{7}$. " L'émergence de la culture de la vigne apparaît finalement dans un contexte de mutations des espaces ruraux québécois qui démarrent dans les années 1950-1960 sous l'impact, d'une part, de la mécanisation et de l'informatisation des exploitations agricoles et, d'autre part, de la diversification des productions agricoles à l'échelle provinciale. Ainsi, « la petite ferme polyvalente et reproduite à des milliers d'exemplaires a cédé sa place à de grandes fermes spécialisées moins nombreuses dans un espace productif qui s'est lui-même diversifié » (Deshaies, 2003). Ces mutations des espaces ruraux annonçant la fin de la ruralité et des fondements traditionnels de la société québécoise (Moreux, 1982) ont été de pair avec un désenchantement de la ville et des rêves que son image liée à la modernité avait générés.

La campagne et notamment les campagnes proches des métropoles, ainsi lissées de leur valeur identitaire, devenues uniformes et ayant perdu de leur singularité, sont devenues des espaces de possibles installations pour des urbains en quête de nature et des lieux d'expérimentations pour des agriculteurs las des prescriptions internationales en matière agricole.

Majoritairement, les viticulteurs rencontrés ${ }^{8}$ sont des urbains issus de catégories socioprofessionnelles fort variées allant de la classe moyenne à la classe supérieure. Selon la taille des domaines (de 1,5 à 20 hectares), les propriétaires s'occupent de leur vignoble soit seul (leur conjoint ayant toujours un emploi à l'extérieur pour un revenu d'appoint), soit ils sont aidés par des employés (œnologues, ouvriers agricoles). Pour les propriétaires appartenant aux catégories socioprofessionnelles les plus élevées (avocats, hommes politiques, etc.), l'activité vitivinicole est une activité supplémentaire et ne constitue pas l'essentiel des revenus individuels. Cette distinction entre « gros » et « petits » viticulteurs révèle, dans les discours, une césure de classes; classes ayant chacune des rapports au territoire viticole et une légitimité différents. Aux plus petits reviendrait la connaissance du travail de la vigne, des sols et les contraintes liés à la pratique «en plein air ».

Ils se perçoivent comme des " gars des champs », ceux qui subissent les rigueurs et les aléas d'un climat fort rigoureux. Finalement, se sont eux les véritables pionniers de cette aventure collective car ils en ont une expérience physique : "nous, on accomplit la tâche de A à Z et on fait que la vigne puisse survivre dans notre climat ». Les " gros » pour leur part, ceux " qui ont de l'argent », "des gens en affaire qui avaient déjà des entreprises et qui disent: moi, je vais démarrer un vignoble mais je vais mettre les moyens techniques pour démarrer », sont considérés comme bien éloignés de la réalité viticole québécoise ("ils connaissent rien, et c'est pas parce qu'on a son nom sur une étiquette qu'on sait faire du vin », "ils en ont fait une entreprise ») et par leur venues tardives (à partir des années 1995), sont soupçonnés d'avoir déstabilisé le marché donnant «plus de misère aux petites entreprises à compétitionner ». On trouve aussi, dans l'échantillon de viticulteurs rencontrés des retraités ou préretraités faisant de l'activité viticole un nouveau projet de vie ou de fin de vie.

Cependant, quelle que soit la catégorie socioprofessionnelle à laquelle ils appartiennent, les motivations qui les ont conduit à la production de vin les réunissent. Ainsi, unanimement, ils font appel au rêve pour expliquer leur choix. Le rêve de «faire du vin », un rêve toujours conditionné

7. Les citations en italiques sont extraites des entretiens.

8. Notre échantillon est composé d'hommes âgés, dans tous les cas, de plus de 40 ans et $30 \%$ sont retraités ou en passe de l'être. $46 \%$ d'entre eux sont issus d'une catégorie socioprofessionnelle moyenne (cadre moyen, technicien), $54 \%$ d'une catégorie socioprofessionnelle supérieure (avocat, entrepreneur, chef d'entreprise). Dans le premier cas, l'activité viticole est souvent exclusive (ou est souhaitée exclusive, à plus ou moins brève échéance) mais le salaire du conjoint dans une autre branche d'activité apparaît et est déclaré comme un salaire d'appoint nécessaire à la survie du ménage. Dans le second cas, l'activité viticole est d'une nature plus récréative et le propriétaire a recours à du personnel, plus ou moins qualifié (vendangeurs, œnologue, maître de chai) soit de façon permanente soit de façon saisonnière pour assurer le bon fonctionnement de la propriété. 
par une passion soit pour cette boisson : "Il y a 19 ans, je suis tombé à genoux devant une bouteille de Bourgogne et ça a commencé. »; "J'ai vu chez un voisin, une très, très belle récolte de Chaunac, ça a été l'amour tout de suite, je suis tombé à genoux devant ça. " Soit pour les paysages viticoles : "Je trouve ça beau, les vignes, les alignements, les rosiers, c'est comme des oasis, c'est la paix. »; "Ce que j'aimerai faire, c'est un vignoble comme en Toscane. " "J'ai voulu ça car c'était beau, c'était pas commun par ici, c'était une envie».

Ce rêve n'est pas «a-topique » mais se réfère au modèle européen, un modèle qui revient de façon récurrente dans les propos et se matérialise sous diverses expressions. D'abord, l'emblème du vin européen est le vin rouge, grands vins d'Italie, Espagne ou France mais aussi vins de pays comme ceux du Roussillon ou du Languedoc. Par cette préférence, les viticulteurs sont tentés par la plantation préférentielle de vignes rouges, plantation souvent revue à la baisse («les rouges, on en rêve tous, mais c'est pas évident ") après quelques malheureuses tentatives ou sur les conseils d'œnologues avertis car «le meilleur vin qu'on fait sur le vignoble ici, c'est le vin blanc et le climat par lui-même se prête plus à faire un vin blanc. Mais le consommateur nous tire aussi vers le rouge avec le French paradox, ça c'est infernal».

Ensuite, tous ont tenté, persisté ou en définitive renoncé, à la culture de cépages nobles : Riesling, Gamay, Cabernet, etc. certains, plus propices, se sont maintenus, les autres abandonnés en désespoir de cause ou sont confinés sur des parcelles "expérimentales » et objets de tous les égards ("j’utilise pour protéger les viniféra des géotextiles, c'est une solution un peu haute technologie, on taille et après on abrite d'une couverture. Ça cô̂te les yeux de la tête mais c'est un peu ma petite parcelle de rêve, quelque chose que je voulais essayer, c'est pas nécessairement rentable».).

Enfin, certains d'entre eux entreprennent de se rapprocher des vignobles européens en jouant, d'une part, sur la densité de pieds à l'hectare pour tenter une augmentation de la qualité («Ici (Montérégie), la densité est de 5500 plants à l'hectare, on rentre dans une densité qualitative alors que dans la région de Dunham, c'est 3200 plans à l'hectare »), la limitation des rendements à l'hectare étant en quelque sorte "naturelle» («le rendement au Québec est de 30 hecto/hectare. On enlève un peu de raisin et on arrive à 25 hecto/hectare. On peut pas faire mieux autrement on va mourir. Dans le Bordelais, les rendements sont de 80 hecto/hectare, dans le Saint-Émilion, 35, on est en dessous. On est en dessous aussi des grands bourgognes »). De façon plus anecdotique, certains « pour le symbole» plantent un pied de rosier en bout de rangée.

\section{DU RÊVE À L'EXPÉRIMENTATION}

"Mais le rêve est une chose et la réalité une autre », la réalité pour les viticulteurs interrogés est le climat québécois «climat froid, climat nordique » impliquant que " faire de la vigne ici est contre nature ». Ainsi, il faut faire contre ou plutôt avec la nature : les froids extrêmes $\left(-38^{\circ} \mathrm{C}\right.$ l'hiver 2003-2004), les gelées de printemps, les étés pluvieux, etc. Ces rigueurs néfastes au bon développement de la vigne obligent les viticulteurs à sans cesse « refaire l'ouvrage ». Ce qui semble acquis (techniques, cépages) peut, à la défaveur d'une saison, être remis en cause. Ainsi, l'ensemble de nos interlocuteurs se désigne comme des pionniers car l'expérience relève de l'aventure et d'une remise en cause continuelle ( "c'est une aventure », «il faut être toujours inventif »).

Se considérant comme des initiateurs, des «constructeurs », leur tâche relève en tout premier lieu de l'expérimentation à tous les niveaux de la filière. En effet, ils expérimentent les cépages afin de déterminer celui ou ceux qui résisteront le mieux aux grands froids. En contact avec des viticulteurs et des centres universitaires européens (Suisse, Allemagne) et américains (Ontario et Minnesota), ils «testent » à partir des conseils glanés lors de voyage ou sur les sites viticoles d'Internet. Chaque cépage est planté sur une ou plusieurs parcelles et soigneusement suivi pendant plusieurs saisons. Cette phase d'expérimentation explique bien entendu la variété importante de cépages plantés dans chaque propriété.

Ils expérimentent ensuite les systèmes de protection de la vigne contre le froid. Si le buttage est dominant (« on enterre les vignes en hiver et on fait l'opération contraire en été. C'est un peu plus 
d'ouvrage »), d'autres techniques, plus ou moins sophistiquées et dépendant de la taille et de la fortune de l'exploitations, sont testées comme le paillage («depuis quelques années, on commence à travailler avec de la paille. On fait un gros paillage, mais un gros paillage, on couvre le plant»), les canons à neige ("depuis quelques années, il y a moins de neige, la neige protége la vigne, 70 à 90 centimètres, c'est bien ", "la neige, pour nous, c'est l'idéal, un mètre à partir de novembre ») et l'utilisation d'hélicoptères pour rabattre l'air chaud et prévenir les gelées du printemps.

Ils expérimentent aussi les diverses techniques d'assemblage pour adoucir le vin, atténuer l'acidité des hybrides résistants souvent issus de vignes sauvages : "Le Frontenac, il a un fort potentiel viticole. C'est comme une vigne sauvage, son problème c'est l'acidité, on parle de $13 \mathrm{~g}$ par litre en acide sulfurique à la vendange. Le Maréchal Foch est à $7 \mathrm{~g}$ par litre. Un acide malique débile mais un taux de sucre intéressant donc au niveau fermentation alcoolique, y a pas de problème, y aurait du sucre pour faire un vin charpenté. »

L'ensemble de ces expérimentations peut se mesurer à travers la grande variété des types de vins produits. Ainsi, pour l'ensemble des vingt vignobles visités, on compte 14 sortes de vins rosés différents, 38 sortes de vins blancs secs, 42 sortes de vins rouges, 22 types d'apéritifs, vins fortifiés (type porto) et digestifs et enfin 8 sortes de vins de glace et de vendanges tardives.

L'expérimentation la plus réussie est, pour l'ensemble de nos interlocuteurs, celle relative à la production du vin de glace même si tous n'en produisent pas. Sur le modèle de l'Allemagne et plus récemment de l'Ontario, les premières expériences reviennent aux propriétaires du domaine de l'Orpailleur (Estrie). Ce vin (issu du cépage Vidal), aux arômes puissants et complexes, est produit à partir de raisins surmaturés d'octobre à janvier, ce qui entraîne une dessiccation des baies et donne un jus à la fois plus riche et plus concentré. Quand $100 \mathrm{~kg}$ de raisins donnent normalement de 80 à 85 litres de moût, ce volume n'est plus que 12 à 15 litres lors de la pressée à $-12{ }^{\circ} \mathrm{C}$. La faible production explique son prix élevé, environ 40 dollars les $200 \mathrm{ml}$ («à ce prix, c'est un flacon de parfum»).

Il représente en fin de compte, la meilleure revanche sur la nature ( $i l$ fait de notre faiblesse de l'hiver une force ", "notre climat est difficile mais ça fait quelque chose d'extraordinaire. On profite de la difficulté pour faire quelque chose qui sort du commun») et devient de ce fait, pour les viticulteurs, le "vin emblématique du Québec ». Malgré tout, sa production est considérée comme "frustrante, c'est du vin que l'on fait en toute petite quantité mais c'est vraiment d'une qualité assez surprenante ». Cette production et les conditions de son élaboration engendrent aussi quelques préoccupations. D’abord, depuis trois ou quatre années, les conditions météorologiques ont été peu favorables puisque les températures se sont radoucies pendant la période de récolte. En effet, il aura fallu «attendre jusqu'à la dernière limite pour qu'il y ait 4 journées consécutives entre -8 et $-12{ }^{\circ} \mathrm{C}$, ça c'est le stress de l'hiver ». Ainsi, pour palier aux récentes variations de température durant la journée, les viticulteurs prévoient de récolter la nuit «comme en Ontario, cela aura plus de charme ». Ensuite, une autre crainte est relative, d'une part, aux fortes productions de vin de glace par l'Ontario : ( ils vont inonder le marché et si, nous aussi, on produit trop, on risque de tuer la poule aux œufs d'or ») et, d'autre part, à la concurrence déloyale de certains producteurs américains qui plutôt que de faire du vin de glace dans le respect des façons de faire héritées de l'Allemagne, produisent $\mathrm{du}$ "vin de glacière » " $i l s$ font $d u$ vin de glace dans les frigos. Ils mettent leurs raisins dans des frigos; ils le mettent sur les étiquettes, ils ne s'en cachent pas ", "bientôt, il y aura plusieurs millions de caisses par année. Ils vont entrer sur le marché et le casser. Il faut qu'il y ait des normes »).

Une autre tentative du même type est initiée notamment par Charles Crawford (domaine Pinacle) et concerne le cidre de glace. Issu de longues et difficiles expérimentations et empruntant les techniques opératoires du vin de glace, ce produit apparaît, aujourd'hui, comme le plus à même pour représenter la singularité du Québec car il y est né et en ce sens « est un pur produit du terroir québécois » et de plus, il est issu de la rencontre de ses «deux icônes : la pomme et le froid » (Lord, 2005). 


\section{DE L’EXPÉRIMENTATION À UNE VISION PROSPECTIVE}

Si le chemin parcouru, en un peu plus de vingt ans, a permis que le rêve de produire du vin se matérialise, en fin de compte les résultats obtenus sont encourageants ("on ne rattrapera pas une culture millénaire mais on s'améliore sacrément. C'est ce chemin là que nous faisons, lentement mais nous avançons ») et typique du lieu ("ici, on fait autre chose qu'ailleurs, ça c'est notre force. De toute manière, on peut pas reproduire la Californie au Québec»).

Cette typicité recèle goût du terroir et doit sa singularité au climat qui oblige à planter des cépages résistants et maturant dans un temps court («ici, on parle de 150 jours sans gel, peu de cépages mûrissent à temps. Les goûts demeurent quelque peu sauvages... la moitié de leur sang est sauvage alors les vins gardent des caractéristiques sauvages, sans que ce soit le lambrusca, c'est quand même sauvage") mais permettant la fabrication de vins différents et hors des standards : "Les palais européens ne les reconnaissent pas, cela ne veut pas dire que c'est pas bon, seulement que ce n'est pas typique du goût international. »; "Le goût des vins québécois va percer, les gens aimeront en boire car ils proposent autre chose, d'ailleurs, de loin, du froid et de la neige, on doit compter là-dessus. »

Dès 1987, les premiers viticulteurs se constituent en association. L'association des vignerons du Québec a alors pour ambition, dès cette époque, de regrouper les volontés des uns et des autres par la mise en place d'actions communes pour envisager un avenir prometteur.

Progressivement, l'association deviendra l'organisme représentatif des intérêts des vignerons mais aussi de défense de ces mêmes intérêts. Nos interlocuteurs voient dans cette association tant « une place de rencontre pour échanger nos problèmes et trouver des solutions ensemble » qu'un formidable outil les rapprochant des pouvoirs publics et notamment de la très puissante Société des Alcools du Québec (SAQ) ("l'association nous permet d'être plus forts et de nous faire reconnaître »).

Le discours tenu par l'association et notamment par son président, M. Belisle (domaine la Roche des Brises) concentre les arguments sur la dynamique territoriale qu'entraînerait une valorisation de cette activité. En effet, face aux problèmes des espaces ruraux, l'activité viticole est présentée comme une activité à haute valeur ajoutée. Elle a, et aurait, si son développement était accompagné par les pouvoirs publics, un fort impact économique : "L'État n'a jamais réfléchi à la dimension économique d'une industrie du vin contrairement à l'Ontario ou la Colombie britannique. Une industrie extraordinaire pour la création d'emplois, l'agro-tourisme, permettant une hausse de la valeur des terres agricoles et la structuration des régions et qui apporte une manne sur le plan fiscal. " Peu ou pas aidée par les pouvoirs publics jusqu'à présent, l'association tente de faire la preuve des avantages du développement et de la structuration de la filière.

Ainsi, une des façons de sortir du flou des expérimentations est, pour les viticulteurs appartenant à l'association, de mettre en place des normes de qualité. Les normes proposées concernent les méthodes et les savoirs faire en matière de vinification notamment pour la production du vin de glace. En effet, pour se protéger de la concurrence et notamment des techniques artificielles (vins de glacières, utilisation non contrôlée de méthodes de bonification, etc.), un label «vin du Québec » garantirait, d'une part, la rigueur des méthodes employés et, d'autre part l'origine, facilitant ainsi le choix des consommateurs qui auraient l'assurance de consommer un vin produit selon des procédés établis et issus d'une origine garantie ( $i$ ici, on est pas trop furieux de se faire appeler vin $d u$ terroir parce qu'on fait $d u$ vin mais le terroir, ça compte mais bon. Il faut mieux s'appeler vin $d u$ Québec car quand on achète du Bourgogne, c'est un endroit. Le terroir c'est rien. À la SAQ, ils nous ont mis dans vins du terroir avec l'hydromel, les confitures, ça fait un méli-mélo »).

Mais ces normes, d'après nos interlocuteurs, et ce contrairement aux normes du vieux continent et notamment de la France, doivent être adaptables et révisables à tout moment pour « garder la liberté qui est nécessaire pour faire du bon vin », "faire du vin est une expression de soi, alors si on dit faites $d u$ vin selon ces instructions, vous n'êtes plus vous-même », "on doit forcer la qualité mais on ne rompt pas l'inventivité au niveau des vignobles». Toute idée d'imposer des normes sur les cépages ou sur les assemblages est rejetée car compte tenu de la jeunesse du vignoble et malgré les nombreuses expérimentations, l'époque est encore aux essais : "On n'est pas au bout, il faut encore 
chercher des nouveaux cépages qui s'adaptent bien ici. »; "Déjà, il y a des progrès avec les cépages qui viennent du Minnesota. "; "Nous mettre des normes au niveau des cépages, des rendements, ça nous mettrait des barrières dont on n'a pas besoin, on a encore besoin d'expérimenter. »

Une autre tâche de l'association et de chacun de ses membres est de faire connaître le vin aux Québécois. L'engouement pour le vin au Québec est récent et, de l'avis général, les consommateurs débutants ont encore peu de repères : "La connaissance des vins, j'en ai vu tantôt, ils reconnaissent pas les goûts dans les vins. Un consommateur moyen français, j'imagine, il est capable au moins de reconnaître certains vins. Ici, je leur dis: "S'il y a de la cannelle dans la tarte aux pommes, vous la reconnaissez. Pourquoi? Car depuis l'âge de 12 ans, vous mangez de la tarte aux pommes et la connexion se fait." Dans le vin, c'est la même chose, à force de goûter, on identifie les goûts. »

L'apprentissage du goût du vin passe aussi par la connaissance des vins québécois. Ainsi, diverses actions ont été mises en œuvre. D’abord, une route des vins est créée en 1989 et jalonnée de panneaux indicateurs à partir de 1993. Elle draine des touristes québécois mais aussi ontariens, californiens, etc. Elle permet aux propriétaires d'écouler la presque majorité de leur production. Les viticulteurs participent ensuite à de nombreuses manifestations durant la période estivale ("par ce biais, on se fait connaître ») : Festival de la Gastronomie à Québec, la clé des champs à Dunham, le Festival des fromages à Warwick, le Festival des vins de Terrebonne, la fête des vendanges à Magog, le week-end gourmand de Rougemont, etc. La présence dans les manifestations est un moyen de se faire connaître et de faire reconnaître ses vins : "J'ai maintenant des clients fidèles que j'ai rencontrés lors de ces événements. »

En effet, la première difficulté avancée par les viticulteurs est la commercialisation de leur production "la plus grande difficulté ici, ce n'est pas le climat mais la mise en marché ». Le premier des combats mené est une libéralisation de la vente par la SAQ, «avoir un présentoir correct pour nos vins » et " pouvoir vendre chez les dépanneurs ${ }^{9}$ ».

\section{Conclusion}

La route est longue et difficile mais malgré les contraintes et les obligations, les viticulteurs ne manquent pas de bonne volonté : «Pour cela encore, nous sommes les pionniers. » La reconnaissance et la légitimité des vins québécois semblent passer par une bataille nationale pour enfin pouvoir avoir lieu sur le marché mondial. Ainsi, une fois toutes ces premières batailles menées, les vignerons espèrent enfin l'achèvement d'une première étape avant qu'une seconde ne surgissent "faire du marketing pour prouver que notre vin est particulier, qu'il ne pourrait être fait nulle part ailleurs ». Cela passera par un travail tout nouveau auquel se heurtent bon nombre de viticulteurs européens, la construction d'une image simple et permettant d'authentifier à Montréal, Moscou, Pékin, Los Angeles ou ailleurs le vin québécois, de le reconnaître comme singulier par son goût ou son origine. Les viticulteurs, même les plus confiants, ne semblent pas rêver de concurrencer les grands vins aux fortes productions mais souhaiteraient créer des niches « pour des connaisseurs, des gens qui cherchent un petit plus ", "ceux qui ne veulent pas faire comme les autres ». Pourquoi le vin québécois ne serait pas un des vins qui permettra dans l'avenir une forme de distinction des élites internationales?

\section{Bibliographie}

Augustin (J.-P.), 1993. - «Civilisation du vin et tourisme culturel. Les routes du vin au Canada », Études Canadiennes/Canadian Studies, n 35, 1993, p. 245-256.

9. Petites épiceries aux heures d'ouverture étendues. 
Association des Vignerons du QuéBec, 2005. « Meilleure visibilité de nos vins dans les SAQ », [www. vignerons-du-quebec.com/evenements-nouvelles.php].

Balandier (G.), 1983. - « Préface », p. 7-9, dans Ferrarotti (F.), Histoire et histoires de vie. Méthode biographique dans les sciences sociales, Paris, Librairie des Méridiens, 195 p.

De Koninck (R.), 1992. - « Le vin et la vigne au Québec. Bon goût et ténacité vigneronne », Cahiers de Géographie du Québec, vol. 37, n 100, p. 79-111.

De Koninck (R.), 1993. - « La viticulture au Québec : du folklore ou une affaire », Études Canadiennes/ Canadian Studies, n 35, 1993, p. 281-299.

Deshaies (L.), 2003. - "Une co-construction terroir-territoire-identité. Le cas de Dunham en Estrie ", p. 215-231, dans Lasserre (F.), Lechaume (A.) (dir.), Le territoire pensé. Géographie des représentations territoriales, Montréal, Presses universitaires du Québec, 328 p.

Deshaies (L.), Dubois (J.-M.) (dir.), 1993. - «Vins et vignobles artisanaux au Québec », Géographes, nº4, numéro spécial, $110 \mathrm{p}$.

Deshaies (L.), Dubois (J.-M.), 1997a. - Guide des Vignobles du Québec, PUL-IG, Québec, 297 p.

—, 1997b. - Une viti-viniculture québécoise méconnue. Dialogue scientifique, vol. 1, n 3, p. 13-16.

Dion (R.), 1990. - Le paysage et la vigne. Essais de géographie historique. Paris, Payot, 288 p.

Dubois (J.-M.) et Deshaies (L.), 1997. - Études d'opportunité sur le développement de l'industrie viti-vinicole dans le Haut-Richelieu, Comité de travail communautaire du Haut-Richelieu, Saint-Jean, 1997, 55 p.

Dubois (J.-M.), 1996. - « La viti-viniculture québécoise : entre "froidure" et vente au vignoble », Annales de géographie, nº 592, p. 603-619.

Environnement Canada, 1998. - «Statistiques climatiques", [http://weatheroffice.ec.gc.ca/canada_ f.html].

Hope-Ross, (P.), 2006. - De la vigne à la coupe : la production de raisins et du vin au Canada, Statistique Canada (Ottawa), 25 p.

LASSERRe (F.), 2001. - «L'essor du vignoble au Québec. Histoire de climats et de goûts », Cybergéo, Revue européenne de géographie (Paris), n 190, p. 1-12.

Lond (D.), 2005. - «Cidre de glace. Né au Québec, il conquiert le monde... », Le Devoir, Montréal (16 mars 2005), p. 12, [http://www.ledevoir.com/index.html].

Manivet (B.), 2005. - La dynamique du processus de choix d'une bouteille de vin chez les consommateurs québécois, Essai de maîtrise en administration des affaires (MBA), Université Laval, Québec, 128 p.

Masson (G.), 1983 - Vigne et vin au Canada : manuel de vinification, Niagara on the Lake, Georges Masson éditeur, $236 \mathrm{p}$.

MÉTÉo France, 1999. «Climat par département », [www.meteofrance.com].

Mapaq (ministère de L’Agriculture, des PêCheries et de l'Alimentation du Québec), 2000. - L'industrie de la vigne au Québec, Québec, 52 p.

Moreux (C.), 1982. - Donceville en Québec. La modernisation d'une tradition, Montréal, Presses Universitaires de Montréal, $454 \mathrm{p}$.

Organisation INTERNATIONALE DE LA VIGNE ET Du VIN (OIV), 2003. Situation et Statistiques du secteur vitivinicole mondial, [http://news.reseau-concept.net/pls/news/p_entree?i_sid=Ei_type_edition_id=17597Ei_section_id=Ei_lang=33].

Roudié (P.), 1989 - «Vignes et vins des tropiques : cultures et productions de riches en pays pauvres », dans Pauvreté et développement dans les pays tropicaux. Hommage à Guy Lasserre, Talence, Presses Universitaires de Bordeaux, p. 276-288.

Statistiques Canada, 2006. «Wine and vineyards in Canada », [www.statscan.ca].

Velasco-Graciet (H.), Hinnewinkel (J.-C.), 2005. - « Les vins de l'hémisphère sud et la mondialisation de la planète viticole », Les Cahiers d'Outre Mer, Bordeaux, n² 231-232, p. 267-280.

VITISPHÈRE, 2006. «Économie», [http://www.vitisphere.com/home_economie.php].

Cet article a été reçu le 27 juillet 2006 et définitivement accepté le 14 décembre 2006. 